\title{
SUPPORTING INFORMATION \\ Selective Lanthanide Sensing with Gold Nanoparticles and \\ Hydroxypyridinone Chelators
}

Roger M. Pallares, $\uparrow$ Korey P. Carter,$\uparrow$ Steven Zeltmann,$\ddagger$ Toni Tratnjek, $\uparrow$ Andrew M. Minor,

and Rebecca J. Abergel $*, \dagger, \S$

†Chemical Sciences Division, Lawrence Berkeley National Laboratory, Berkeley, CA, 94720, USA.

\$National Center for Electron Microscopy, Molecular Foundry, Lawrence Berkeley

National Laboratory, Berkeley, CA 94720, USA; Department of Materials Science and

Engineering, University of California, Berkeley, CA, 94720, CA

$\S$ Department of Nuclear Engineering, University of California, Berkeley, CA, 94720, USA

*E-mail: abergel@ berkeley.edu

\section{TABLE OF CONTENTS}

Table S1. Stability constants used in the data refinement or determined in this study . S2

Figure S1. Cyclic voltammetry of Au3+ and 3,4,3-LI(1,2,-HOPO) at pH 5.9 ................... S3

Figure S2. Speciation diagram for Au3+ and 3,4,3-LI(1,2-HOPO)................................S4

Figure S3. Size distribution of AuNPs................................................................... S5

Figure S4. The AuNPs were multiply-twinned crystals. .................................................. S6

Figure S5. Elemental mapping of AuNPs grown with 3,4,3-LI(1,2-HOPO). .................... S7

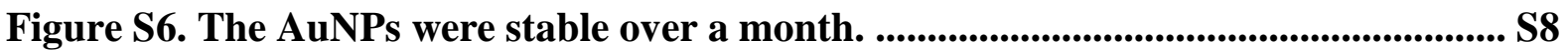

Table S2. Characterization of AuNPs ................................................................................... S9

Figure S7. Characterization of 3,4,3-LI(1,2-HOPO) on AuNP surface........................ S10

Figure S8. 3,4,3-LI(1,2-HOPO) on AuNP surface preserved selectivity towards f-block

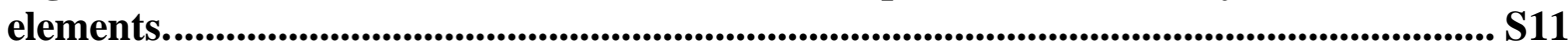

Table S3. Quantification of reference sample ............................................................ S12

Supporting Information References......................................................................... S13 
Table S1. Stability constants used in the data refinement or determined in this study

\begin{tabular}{cccc}
\hline Species a & $\boldsymbol{m}, \boldsymbol{l}, \boldsymbol{c}, \boldsymbol{h}$ & $\log \boldsymbol{\beta}$ & Reference \\
\hline$[\mathrm{OH}]-$ & $0,0,0,-1$ & -13.76 & 1 \\
{$[\mathrm{LH}]_{3-}$} & $0,1,0,1$ & 6.64 & 2 \\
{$\left[\mathrm{LH}_{2}\right]_{2-}$} & $0,1,0,2$ & 12.32 & 2 \\
{$\left[\mathrm{LH}_{3}\right]-$} & $0,1,0,3$ & 17.33 & 2 \\
{$\left[\mathrm{LH}_{4}\right]$} & $0,1,0,4$ & 21.20 & 2 \\
{$\left[\mathrm{AuCl}_{3}(\mathrm{OH})\right]-$} & $1,0,3,-1$ & -5.87 & 3 \\
{$\left[\mathrm{AuCl}_{2}(\mathrm{OH})_{2}\right]-$} & $1,0,2,-2$ & -12.74 & 3 \\
{$\left[\mathrm{AuCl}(\mathrm{OH})_{3}\right]-$} & $1,0,1,-3$ & -20.51 & 3 \\
{$[\mathrm{AuL}]-$} & $1,1,0,0$ & $16.03 \pm 0.04$ & This study \\
{$[\mathrm{AuLH}]$} & $1,1,0,1$ & $17.98 \pm 0.04$ & This study \\
\hline
\end{tabular}

a Where $\mathrm{L}$ is $3,4,3-\mathrm{LI}(1,2-\mathrm{HOPO})$. 


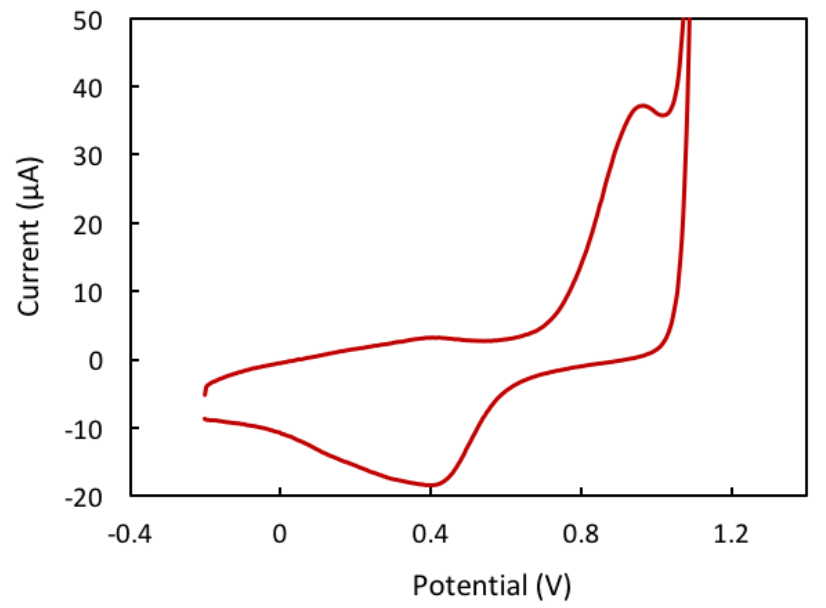

Figure S1. Cyclic voltammetry of Au3+ and 3,4,3-LI(1,2,-HOPO) at pH 5.9 . The measurements were performed with a screen-printed gold electrode, and a silver chloride $\left(\mathrm{Ag}_{+} / \mathrm{AgCl}\right)$ reference electrode at $50 \mathrm{mV} / \mathrm{s}$. 


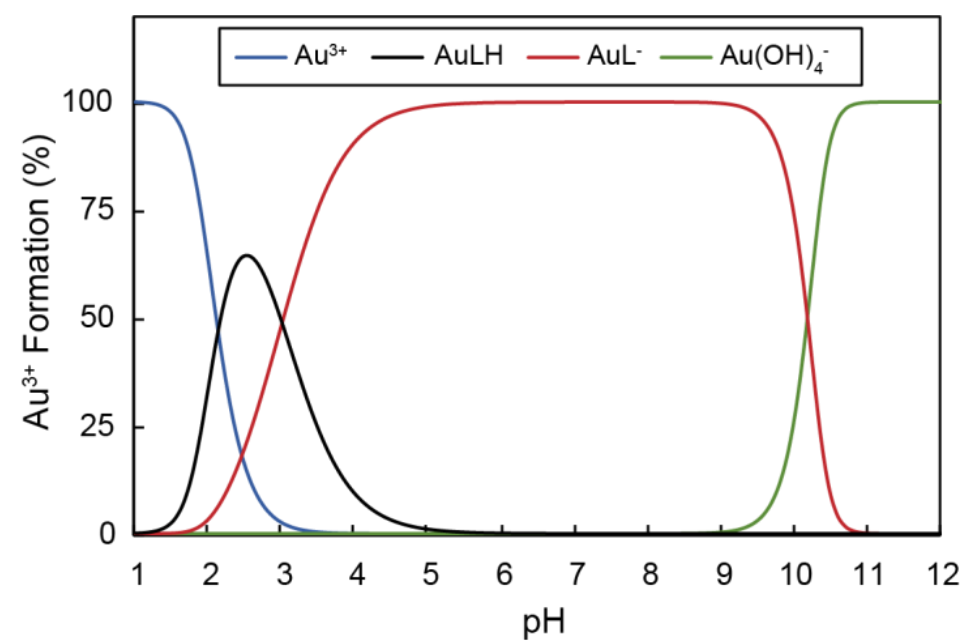

Figure S2. Speciation diagram for Au3+ and 3,4,3-LI(1,2-HOPO). The ligand is written as L. The concentration of $\mathrm{Au}_{3}+$ and chelate was $400 \mu \mathrm{M}$. The stability constants used for the calculations are described in Table S1. 


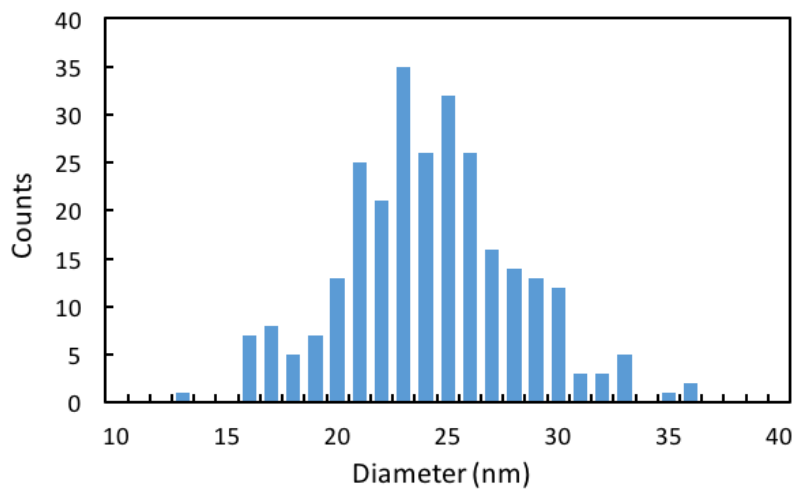

Figure S3. Size distribution of AuNPs. Diameter of AuNPs grown with 3,4,3-LI(1,2HOPO) at $\mathrm{pH} 4.9$. 


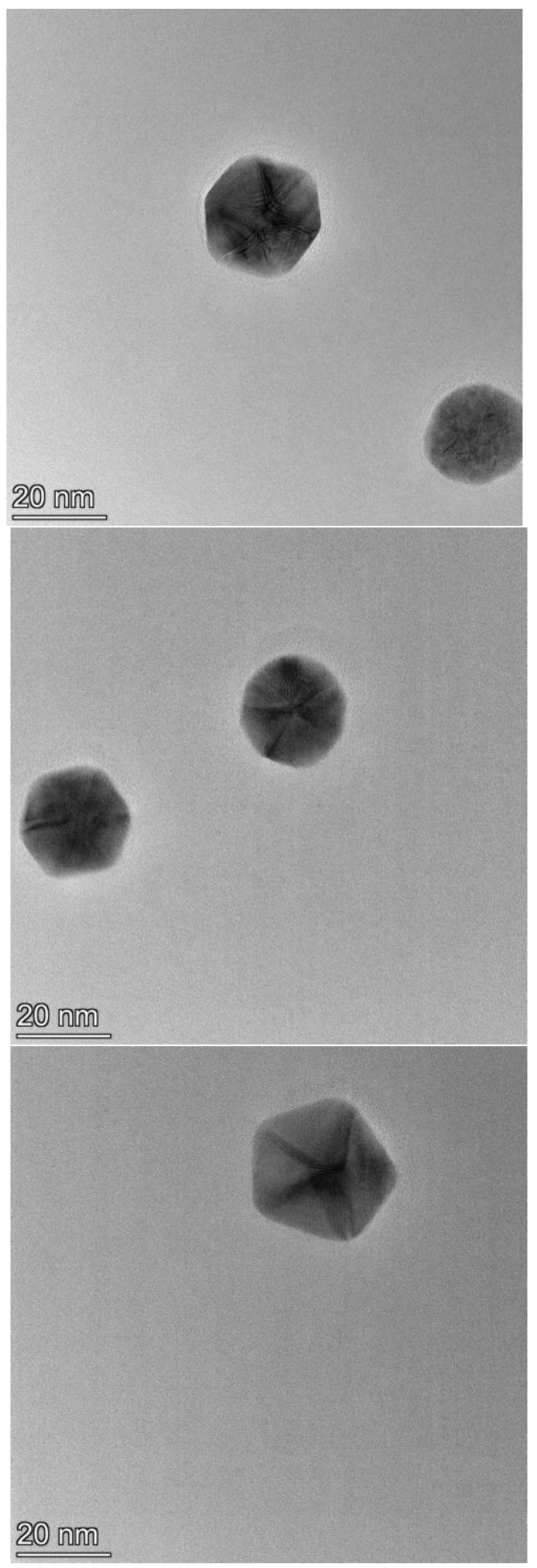

Figure S4. The AuNPs were multiply-twinned crystals. Representative HRTEM images of AuNPs, which were predominantly polycrystalline with decahedron and icosahedron shapes. 


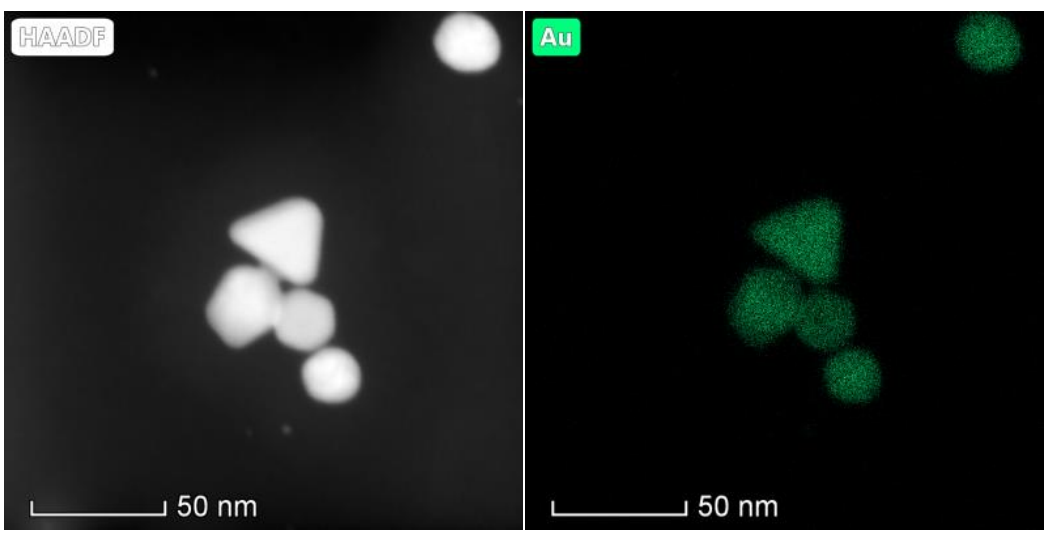

Figure S5. Elemental mapping of AuNPs grown with 3,4,3-LI(1,2-HOPO). AuNPs were simultaneously studied with HAADF (left image) and EDS (right image) modes of a STEM. 


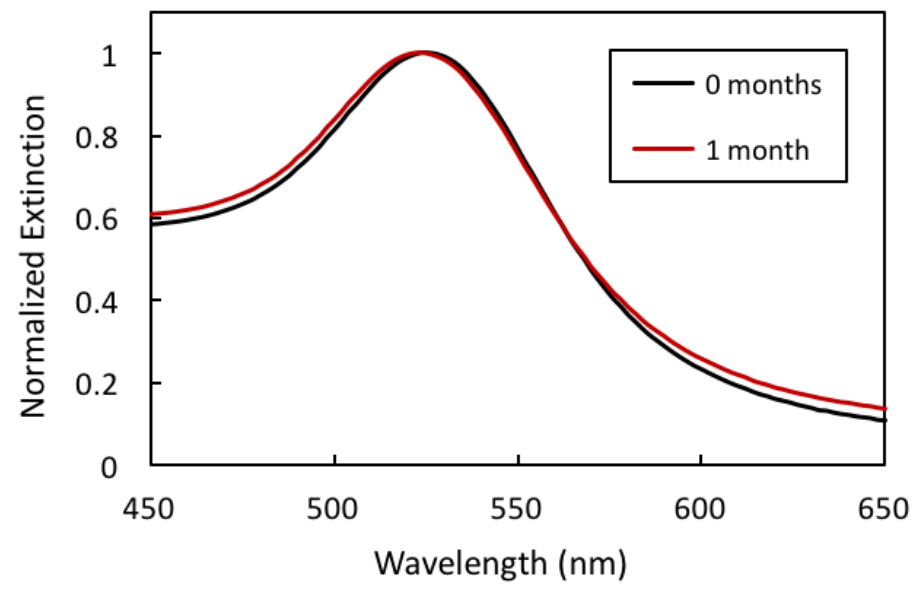

Figure S6. The AuNPs were stable over a month. UV-Vis spectra of washed AuNPs immediately after synthesis and after 1 month. 
Table S2. Characterization of AuNPs

\begin{tabular}{lc}
\hline Nanoparticle diameter 1 & $24 \pm 4 \mathrm{~nm}$ \\
Hydrodynamic diameter 2 & $24.4 \pm 0.1 \mathrm{~nm}$ \\
Polydispersity2 & $0.099 \pm 0.010$ \\
Zeta potential & $-22.9 \pm 2.6 \mathrm{mV}$ \\
LSP max & $526 \mathrm{~nm}$ \\
\hline
\end{tabular}

1measured by scanning transmission electron microscopy.

2measured by dynamic light scattering. 

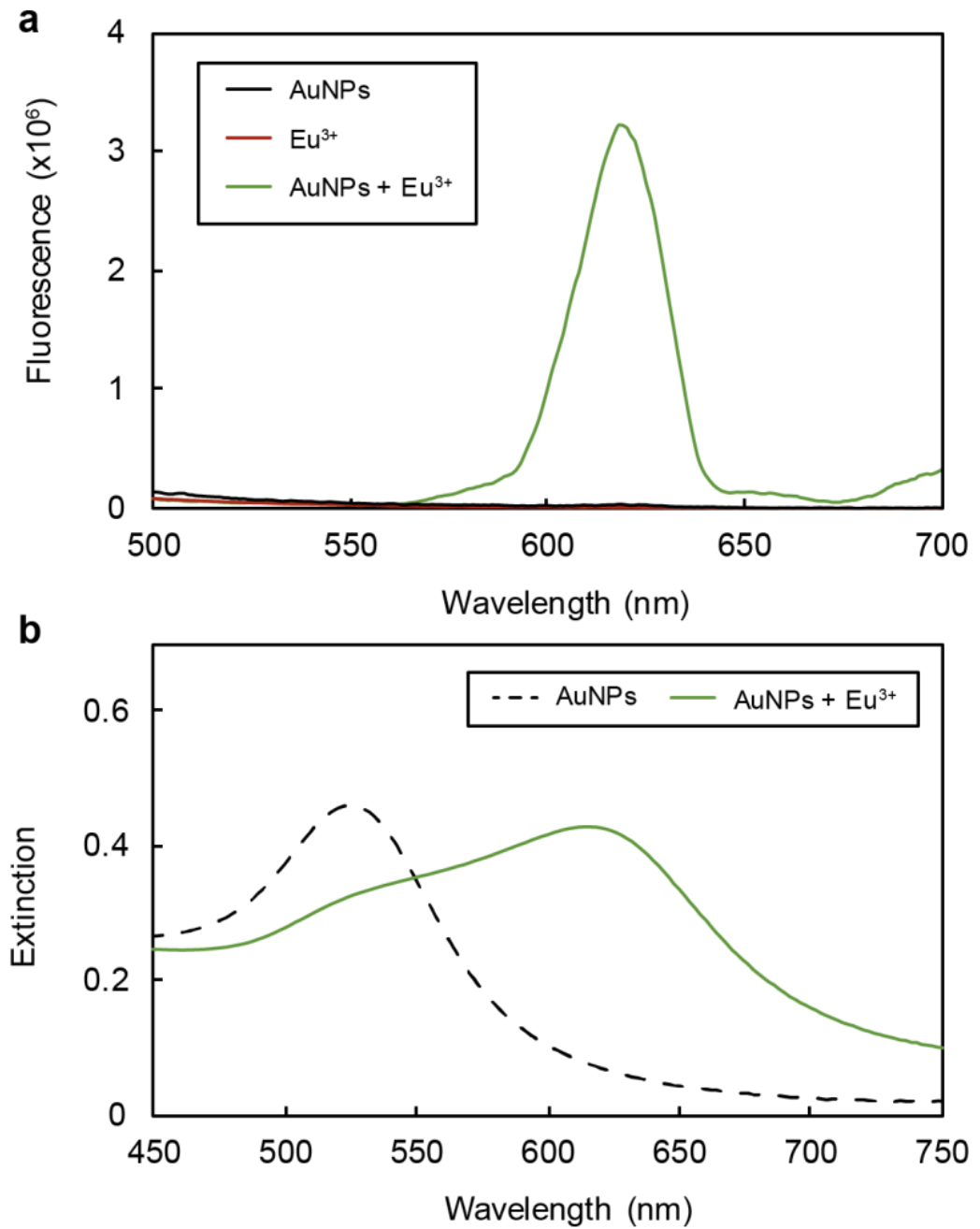

Figure S7. Characterization of 3,4,3-LI(1,2-HOPO) on AuNP surface. (a) Emission of washed AuNPs, Eu3+, and mixture of washed AuNPs and Eu3+ after excitation at $315 \mathrm{~nm}$. (b) UV-Vis spectrum of solution with washed AuNPs and Eu3+. The pH of the solution was kept at 6.0 with MES buffer $(0.1 \mathrm{M})$. The concentration of Eu3+ was $100 \mu \mathrm{M}$ for all solutions. 


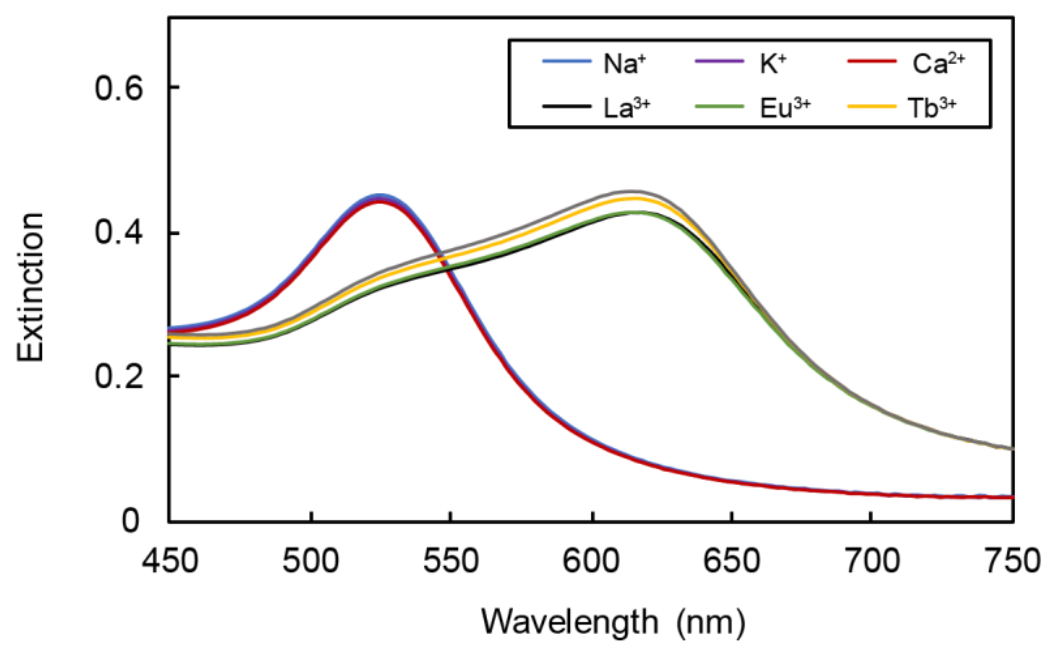

Figure S8. 3,4,3-LI(1,2-HOPO) on AuNP surface preserved selectivity towards fblock elements. UV-Vis spectra of AuNPs with different cations $(10 \mu \mathrm{M})$. The $\mathrm{pH}$ of the solutions was kept at 6.0 with MES buffer $(0.1 \mathrm{M})$. 
Table S3. Quantification of reference sample

\begin{tabular}{cccc}
\hline Sample & $\begin{array}{c}\text { Original } \\
\text { Concentration }\end{array}$ & $\begin{array}{c}\text { Concentration } \\
\text { Quantified }\end{array}$ & Accuracy \\
\hline $\mathrm{EuCl}_{3}$ & $250 \mu \mathrm{M}$ & $284 \pm 8 \mu \mathrm{M}$ & $87 \%$ \\
\hline
\end{tabular}




\section{Supporting Information References}

(1) Bacher, F.; Dömötör, O.; Chugunova, A.; Nagy, N. V.; Filipović, L.; Radulović, S.; Enyedy, É. A.; Arion, V. B. Strong effect of copper(ii) coordination on antiproliferative activity of thiosemicarbazone-piperazine and thiosemicarbazone-morpholine hybrids. Dalton Trans. 2015, 44 (19), 9071-9090.

(2) Abergel, R. J.; D’Aléo, A.; Ng Pak Leung, C.; Shuh, D. K.; Raymond, K. N. Using the Antenna Effect as a Spectroscopic Tool: Photophysics and Solution Thermodynamics of the Model Luminescent Hydroxypyridonate Complex [EuIII(3,4,3-LI(1,2-HOPO))]-. Inorg. Chem. 2009, 48 (23), 10868-10870.

(3) Chateau, H.; Gadet, M.-C.; Pouradier, J. Électrochimie des sels d'or. J. Chim. Phys. 1966, 63, 269-271. 